$\Rightarrow$ NEUROGENESIS

\section{Par for the course}

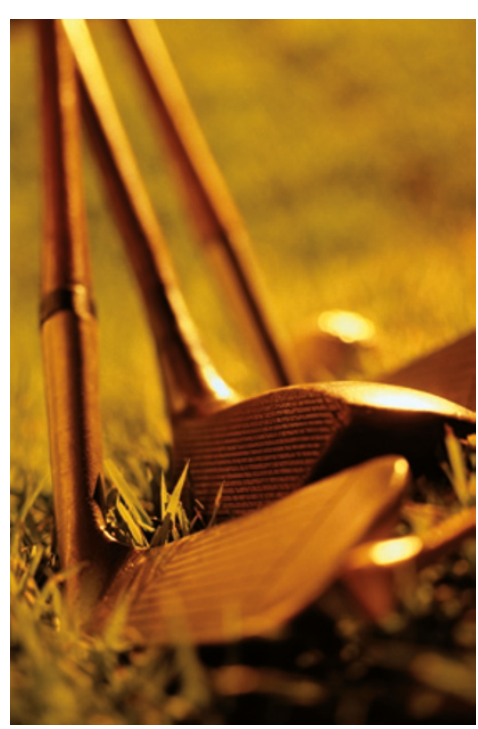

During development, the balance between proliferation and differentiation of progenitor cells ultimately defines the size of the brain. Progenitor cells of the ventricular zone (VZ) show an enrichment of partitioning-defective (Par) proteins - well-established polarity markers - at their apical surface. Götz and colleagues now demonstrate that Parcomplex proteins have a crucial role in maintaining the potential of $\mathrm{VZ}$ progenitor cells to proliferate.

Progenitor cells in the VZ can either continue to proliferate or start differentiating. The molecular basis for the switch from proliferation to differentiation is the subject of intense investigation. Par-complex proteins have a well-characterized role in neuroblast division in Drosophila and have previously been implicated in VZ-progenitor division in vertebrates.

The authors used immunostaining to examine Par-complex-protein expression at various stages of mouse cortical development. Several Parcomplex proteins, including Par3 and Par6, were strongly expressed at embryonal day (E) 12 but significantly lower levels were detected at E14 and E16 - when neurogenesis is reaching its midpoint and proliferation is trailing off.

Next the authors used shorthairpin RNA (shRNA) to knockdown Par3 expression in E12 cortical progenitors in vitro. They found that this decreased the number of cells arising from each progenitor and increased the proportion of differentiated versus non-differentiated daughter cells, indicating that a lack of Par3 results in premature differentiation of progenitor cells. In subsequent in vivo studies in which Par3 expression was reduced by shRNA-containing lentiviral vectors, the transduced progenitor cells began to differentiate soon after transduction and also remained resident in the lower cortical layers, rather than migrating upward with the non-transduced progenitors.

To further characterize the role of Par-complex proteins in VZprogenitors, the authors used retroviral vectors to overexpress Par3 and Par6 both in vitro and in vivo. This resulted in a significant increase in progenitor proliferation and a coincident decrease in differentiation. The authors also showed that these manipulations had no influence on the length of the cell cycle.

Together these results indicate that Par-complex proteins are key for the proliferation potential of VZ progenitor cells, and thus for determining the size and thickness of the cortex.

Craig Nicholson

ORIGINAL RESEARCH PAPER Costa, M. R., Wen, G., Lepier, A., Schroeder, T. \& Götz, M. Par-complex proteins promote proliferative progenitor divisions in the developing mouse cerebral cortex. Development 21 Nov 2007 (doi:10.1242/dev.009951) 